\title{
Perfil das prescrições de antimicrobianos de uso restrito em uma Unidade de Terapia Intensiva
}

\author{
Profile of restricted use antimicrobial requirements in na Intensive Care Unit
}

\author{
Perfil de prescripciones de antimicrobianos de uso restringido en una Unidad de Cuidados \\ Intensivos
}

Recebido: 27/05/2021 | Revisado: 10/06/2021 | Aceito: 24/06/2021 | Publicado: 09/07/2021

Gilvania Nascimento Souza

ORCID: https://orcid.org/0000-0002-4715-4088 Centro Universitário UNIAGES, Brasil

E-mail: souza.gilvania@yahoo.com.br

Murilo de Jesus Porto

ORCID: https://orcid.org/0000-0003-2339-8173 Universidade do Estado da Bahia, Brasil

E-mail: murilo.porto@hotmail.com

Juliana Pereira dos Santos

ORCID: https://orcid.org/0000-0002-5046-743X Universidade do Estado da Bahia, Brasil

E-mail: jupereira630@gmail.com

Anderson Leite Freitas

ORCID: https://orcid.org/0000-0003-0494-7923 Universidade Federal de Sergipe, Brasil

E-mail: andersonlfd@hotmail.com

Phydel Palmeira Carvalho

ORCID: https://orcid.org/0000-0001-6229-943X

Universidade do Estado da Bahia, Brasil

E-mail: phydel_ppc@yahoo.com.br

\begin{abstract}
Resumo
Os antimicrobianos são fármacos que agem inibindo o crescimento ou a morte de microrganismos, sendo a segunda classe de medicamentos mais prescrita em hospitais e responsável por 20 a $50 \%$ das despesas hospitalares com medicamentos. Este estudo teve como objetivo analisar o perfil das prescrições de antimicrobianos de uso restrito em uma Unidade de Terapia Intensiva. Trata-se de um estudo farmacoepidemiológico descritivo de delineamento transversal, com abordagem quantitativa de análise estatística de dados secundários do setor de Farmácia do Hospital Universitário de Lagarto/SE (HUL), entre os meses de janeiro a dezembro de 2019, aprovado pelo Comitê de Ética e Pesquisa da Instituição preponente Faculdade AGES (Número do parecer: 3.758.862). Das 329 solicitações de antimicrobianos de uso restrito referentes ao ano de 2019, 41,51\% dos eram pacientes idosos, 52,83\% do sexo masculino, 26,75\% com diagnósticos de sepse e 23,40\% com problemas do trato respiratório. O uso do Carbapenêmico Meropenem (35,56\%), o glicopeptídeos Vancomicina (20,67\%) e a Piperacilina + Tazobactam (19,77\%) foram os medicamentos mais prescritos. Esse resultado pode ser explicado pelo fato de serem antimicrobianos de amplo espectro, utilizado inicialmente como terapia empírica em situações consideradas graves, e depois mantido como meio de se ter mais segurança e uma ampla efetividade contra diversos microrganismos. Subsidiar indicadores epidemiológicos que podem ser utilizados para a melhoria da assistência à saúde, pode proporcionar a eficácia de tratamentos propostos, além de nortear sobre pontos que necessitam de ajuste no fluxo de prescrição e liberação de tais medicamentos.
\end{abstract}

Palavras-chave: Antimicrobianos; Resistência microbiana a medicamentos; Unidade de terapia intensiva.

\begin{abstract}
Antimicrobials are drugs that act by inhibiting the growth or death of microorganisms, being the second most prescribed medication class in hospitals and responsible for 20 to $50 \%$ of hospital expenses with medications. This study aimed to analyze the profile of prescriptions for restricted-use antimicrobials in an Intensive Care Unit. This is a descriptive pharmacoepidemiological study with a cross-sectional design, with a quantitative approach of statistical analysis of secondary data from the Pharmacy sector at the Hospital Universitário de Lagarto / SE (HUL), between the months of January to December 2019, approved by the Ethics and Research of the institution Institut Faculdade AGES (Opinion number: $3,758,862$ ). Of the 329 requests for antimicrobials for restricted use for the year $2019,41.51 \%$ of them were elderly patients, $52.83 \%$ were male, $26.75 \%$ were diagnosed with sepsis and $23.40 \%$ were with respiratory tract problems. The use of Carbapenemic Meropenem (35.56\%), glycopeptides Vancomycin (20.67\%) and Piperacillin + Tazobactam (19.77\%) were the most prescribed drugs. This result can be explained by the fact that they are broadspectrum antimicrobials, initially used as empirical therapy in situations considered serious, and later maintained as a means of having more security and a wide effectiveness against various microorganisms. Subsidizing epidemiological
\end{abstract}


indicators that can be used to improve health care, can provide the efficacy of proposed treatments, in addition to guiding points that need adjustment in the flow of prescription and release of such drugs.

Keywords: Antimicrobials; Microbial drug resistance; Intensive care unit.

\section{Resumen}

Los antimicrobianos son fármacos que actúan inhibiendo el crecimiento o la muerte de microorganismos, siendo la segunda clase de medicamentos más recetada en los hospitales y responsable del 20 al 50\% de los gastos hospitalarios con medicamentos. Este estudio tuvo como objetivo analizar el perfil de las prescripciones de antimicrobianos de uso restringido en una Unidad de Cuidados Intensivos. Se trata de un estudio farmacoepidemiológico descriptivo con un diseño transversal, con abordaje cuantitativo de análisis estadístico de datos secundarios del sector Farmacia del Hospital Universitário de Lagarto / SE (HUL), entre los meses de enero a diciembre de 2019, aprobado por el Comité de Ética e Investigación de la institución Institut Faculdade AGES (Número de dictamen: 3.758.862). De las 329 solicitudes de antimicrobianos de uso restringido para el año 2019, el 41,51\% de los pacientes eran ancianos, el 52,83\% eran hombres, el 26,75\% tenían diagnóstico de sepsis y el 23,40\% tenían problemas de vías respiratorias. El uso de meropenem carbapenémico $(35,56 \%)$, glicopéptidos vancomicina $(20,67 \%)$ y piperacilina + tazobactam $(19,77 \%)$ fueron los fármacos más prescritos. Este resultado puede explicarse por el hecho de que se trata de antimicrobianos de amplio espectro, inicialmente utilizados como terapia empírica en situaciones consideradas graves, y posteriormente mantenidos como un medio para tener más seguridad y una amplia efectividad frente a diversos microorganismos. Subsidiar indicadores epidemiológicos que se pueden utilizar para mejorar la atención de la salud, puede brindar la eficacia de los tratamientos propuestos, además de orientar los puntos que necesitan ajuste en el flujo de prescripción y liberación de dichos medicamentos.

Palabras clave: Antimicrobianos; Resistencia a fármacos microbianos; Unidad de terapia intensiva.

\section{Introdução}

Os antimicrobianos são fármacos que agem inibindo o crescimento ou a morte de microrganismos, sendo a segunda classe de medicamentos mais prescrita em hospitais e responsável por 20 a 50\% das despesas hospitalares com medicamentos. Este amplo uso pode afetar significativamente tanto a microbiota do indivíduo quanto a do ambiente hospitalar (Brasil, 2017). Assim, o aumento do número de infecções hospitalares surge associadas ao uso inadequado dos antimicrobianos, que costumam elevar o período de hospitalização, o índice de morbimortalidade e o custo das internações (Lemos, 2016).

No contexto hospitalar, a maioria das vezes o uso desses medicamentos é feito de modo exacerbado e desnecessário, resultando no aumento da resistência bacteriana e perda da efetividade terapêutica. Apesar do aumento da resistência microbiana não ser algo recente, a qualidade das prescrições é fundamental para manter a efetividade dos antimicrobianos disponíveis, pois, a Unidade de Terapia Intensiva (UTI) é um local onde há uma maior propensão à ocorrência de infecção hospitalar devido ao perfil de atendimento prestado, aos procedimentos invasivos realizados, a constante utilização de antimicrobianos menos seletivos, a realização de procedimentos cirúrgicos e aguardo pós-cirúrgico, uso de drogas vasoativas e supressoras do sistema imunológico, entre outros (Mata Abegg, 2011).

Segundo a Organização Mundial de Saúde (1985), entende-se que uso racional de medicamentos é "quando pacientes recebem medicamentos apropriados para suas condições clínicas, em doses adequadas às suas necessidades individuais, por um período adequado e ao menor custo para si e para a comunidade”. Ainda de acordo com a leitura do trabalho de Lemos (2016), o uso adequado de antimicrobianos tem que ser visto como umas das etapas da segurança do paciente, sendo que métodos restritivos proporcionam controle no seu uso e eficácia a médio e longo prazo, e a educação é um fator importante para a implementação deste.

Segundo Mota (2010) alguns fatores contribuem para que, direta ou indiretamente, os antimicrobianos sejam utilizados de maneira inapropriada em pacientes. Dentre eles, pode-se destacar a errada concepção de que a eficácia de um tratamento está ligada à utilização de antimicrobianos de amplo espectro, o fato do médico prescritor clínico muitas vezes desconhecer exatamente a dosagem, intervalos e diluições adequadas para cada situação clínica, o que contribui para a não efetividade do tratamento e para o aparecimento de reações adversas e resistência bacteriana, além da ausência de uma comissão no ambiente hospitalar voltada para o seu uso racional. 
A avaliação da Dose Diária Definida (DDD) que é a dose de manutenção média presumida por dia para um medicamento usado para sua principal indicação terapêutica em adultos de $70 \mathrm{Kg}$, é a unidade utilizada pelo Conselho Nórdico sobre Medicamentos, pioneiro nos estudos de utilização de medicamentos e é a unidade recomendada pelo Grupo de Estudos de Utilização de Medicamentos da Organização Mundial de Saúde (OMS).

No Brasil, a Gerência de Vigilância e Monitoramento em Serviços de Saúde - GVIMS da Agência Nacional de Vigilância Sanitária (DATASUS - ANVISA) disponibiliza um formulário eletrônico para que seja informado o consumo aproximado dos principais antimicrobianos utilizados nas Unidades de Terapia Intensiva (UTI) ADULTO de todo país.

É importante destacar que a dose diária definida (DDD) é uma unidade de medida e não reflete necessariamente a dose diária recomendada ou prescrita. Os dados de consumo de antimicrobianos apresentados em DDDs apenas dão uma estimativa aproximada do consumo e não uma imagem exata do uso real, mas permite que se avalie as tendências no consumo dessas drogas e faça comparações entre grupos populacionais. Sendo assim, o presente estudo foi desenvolvido com intuito de realizar o levantamento do perfil das prescrições de antimicrobianos de uso restrito em uma Unidade de Terapia Intensiva Adulto de um hospital de ensino, relacionando com o diagnóstico descrito e identificar o tempo de tratamento utilizado.

\section{Metodologia}

Para esse artigo, foi realizada uma revisão de literatura através dos unitermos: antimicrobiano/antibiótico; uso restrito; antibioticoterapia, na base de dados MEDLINE/PubMed, Web of Science, Lilacs e Scopus. Posteriormente, foi realizado um estudo quantitativo descritivo, com delineamento transversal, realizado no setor de Farmácia do Hospital Universitário de Lagarto/SE (HUL), nos meses de janeiro a dezembro de 2019, sob o consentimento da Gerência de Ensino e Pesquisa da instituição.

A farmácia do hospital realiza as distribuições dos medicamentos antimicrobianos através da dispensação em dose individuais de 24 horas, sendo apenas uma unidade central, não possuindo farmácia satélite. Esta é a responsável pela liberação dos antimicrobianos de uso restrito, recebendo as solicitações dos médicos clínicos plantonistas e entregando-as aos médicos infectologistas que compõem o Serviço de Controle de Infecção Hospitalar (SCIH) na Unidade Hospitalar, responsáveis pelo possível ajuste da medicação ou substituição e pela autorização ou não da continuidade da administração. Através desse mapa de controle determina-se a duração do tratamento e assim a liberação pela Farmácia.

A solicitação de antimicrobianos de uso restrito é necessária para os seguintes medicamentos: Ampicilina + Sulbactam; Anfoterina B; Azitromicina (em sua forma farmacêutica injetável); Cefepime; Imipenem; Linezolida; Meropenem; Piperacilina + Tazobactam; Polimixina B; Teiclopanina; Tigeciclina e Vancomicina. Sendo estes definidos pela Comissão de Controle de Infecção Hospitalar (CCIH).

O Hospital Universitário de Lagarto é vinculado como um Hospital porta aberta de médio porte ao Serviço do Sistema Único de Saúde (SUS), gerenciado pela Empresa Brasileira de Serviços Hospitalares (EBSERH), de caráter federal e vinculada a Universidade Federal de Sergipe (UFS), recebendo constantemente universitários e residentes. Conta com uma Unidade de Terapia Intensiva (UTI-Adulto) com 12 leitos (sendo 2 para isolamentos) de acordo o Cadastro Nacional de Estabelecimentos de Saúde - CNES (Brasil, 2020). Atende ao contingente populacional da região Centro-Sul do Estado de Sergipe, sendo seis municípios que integram a Região de Saúde de Lagarto (Lagarto, Simão Dias, Salgado, Riachão do Dantas, Poço Verde e Tobias Barreto) com uma população estimada em cerca de 255 mil habitantes, além de cidades de outras regionais e do estado da Bahia que fazem divisa com o estado.

Para este estudo, foram utilizados como fontes de dados secundários as fichas de Solicitação dos antimicrobianos de uso restrito recebidas pelo setor de Farmácia do Hospital Universitário de Lagarto/SE (HUL) de todos os pacientes internados na Unidade de Terapia Intensiva (UTI) que necessitou de antimicrobianos de uso restrito. Baseado no diagnóstico clínico dos 
pacientes, foram agrupados segundo a Classificação Internacional de Doenças e foram confrontadas pelo guia antimicrobiano e artigos correspondentes a evidências científicas. Levou-se em consideração a dose, via de administração, duração do tratamento, monoterapia ou associação e o antimicrobiano de primeira escolha.

$\mathrm{O}$ instrumento utilizado neste estudo foram as solicitações de antimicrobianos de uso restrito entregues à farmácia no período citado, onde consta os seguintes dados: nome do paciente; idade; sexo; número de prontuário; setor; leito; nome do medicamento solicitado; posologia e duração do tratamento; justificativa de uso; data da solicitação; além do carimbo do médico solicitante e espaço para a avaliação da equipe de Infectologia/Serviço de Controle de Infecção Hospitalar (SCIH). Foram excluídas as fichas: 1) contendo antimicrobianos que não são de uso restrito; 2) que não estavam corretamente preenchidas; 3 ) de anos anteriores ao ano de 2019; 4) as que não eram da Unidade de Terapia Intensiva; 5) e, que não foram avaliadas pelo Serviço de Controle de Infecção Hospitalar (SCIH).

Os pesquisadores extraíram as informações das fichas de Solicitação dos antimicrobianos de uso restrito elaborada e recebida no setor da Farmácia. Por se tratar de coleta de dados secundários, sem utilizar o nome do paciente, através de estudo ambiespectivo das fichas de dispensação de antimicrobianos de uso restrito. Portanto, foi dispensado a necessidade de Termo de Consentimento Livre e Esclarecido (TCLE), aprovado pelo Comitê de Ética e Pesquisa da Instituição preponente Faculdade AGES (CAAE: 25349619.1.0000.8013 - Número do parecer: 3.758.862).

Foram utilizadas 329 solicitações de antimicrobianos de uso restrito relativo ao ano de 2019. Foram analisadas todas as fichas de solicitação dos medicamentos antimicrobianos de uso restrito de todos os pacientes internados na Unidade de Terapia intensiva do Hospital Universitário de Lagarto, preenchidas em Microsoft ${ }^{\circledR}$ Office Excel 2016. A digitação das informações obtidas foi realizada em duplicata e independentemente por dois pesquisadores e confrontada pelo terceiro pesquisador, eliminando assim vieses do processo de tabulação das informações.

A análise estatística foi feita através das variáveis analisadas e correlacionadas. Para o processamento e análise dos dados foi utilizado o Programa Software for Statitics and Data Science - STATA® 16.0. Sendo realizada a distribuição de frequência absoluta e relativa das variáveis estudadas, organizadas em tabelas e dados. Para associações entre as variáveis foram aplicados os testes Qui-quadrado e o teste exato de Fisher e nos casos aplicáveis, a Razão de Chances (Odds Ratio), com intervalo de confiabilidade de $95 \%$, sendo considerado estatisticamente significante o valor $p$ associado menor ou igual a 0,05 ( $\mathrm{p} \leq 0,05$ ).

\section{Resultados}

Fizeram uso de antimicrobianos de uso restrito 106 pacientes internados na UTI entre 01 de janeiro a 31 de dezembro de 2019, totalizando 329 solicitações de antimicrobianos, sendo uma média de 3,10 antimicrobianos por paciente. Houve uma discreta diferença entre os sexos, sendo $56(52,83 \%)$ pacientes masculinos e $50(47,17 \%)$ pacientes femininos. A maior parte dos pacientes eram idosos, sendo 41,51\% com idade entre 70 e 89, com uma média de 61,09 anos (Tabela 1). 
Tabela 1. Variáveis Sociodemográficas.

\begin{tabular}{lcc}
\hline Sexo & $\mathbf{n}$ & $\boldsymbol{\%}$ \\
\hline Feminino & 50 & 47,17 \\
\hline Masculino & 56 & 52,83 \\
\hline Faixa etária & & \\
\hline 18 a 29 & 10 & 9,44 \\
\hline 30 a 49 & 16 & 15,09 \\
\hline 50 a 69 & 35 & 33,02 \\
\hline 70 a 89 & 44 & 41,51 \\
\hline acima de 90 & 1 & 0,94 \\
\hline
\end{tabular}

Fonte: Autores (2021).

Dos medicamentos analisados desse estudo, o mais solicitado foi o carbapenêmico meropenem. Das 329 solicitações, $117(35,57 \%)$ foram deste medicamento. A segunda classe mais solicitada foram os glicopeptídeos, mais precisamente a vancomicina, com $27,97 \%$, seguido pelas penicilinas $21,28 \%$, polimixinas 7,29\%, cefalosporinas 4,26\%, macrolídeos $2,73 \%$, glicilcilinas $0,6 \%$ e o de menor prevalência (0,3\%) aparecendo apenas uma vez nas solicitações foi a oxazolidinona (Tabela 2 ).

Tabela 2. Uso dos Antimicrobianos de uso restrito solicitados na UTI - Lagarto, 2019.

\begin{tabular}{lcc}
\hline & $\mathbf{n}$ & $\mathbf{\%}$ \\
\hline Meropenem & 117 & 35,56 \\
\hline Vancomicina & 68 & 20,67 \\
\hline Piperacilina + tazobactam & 65 & 19,77 \\
\hline Polimixina B & 24 & 7,29 \\
\hline Teicoplanina & 24 & 7,29 \\
\hline Cefepima & 14 & 4,26 \\
\hline Azitromicina & 9 & 2,74 \\
\hline Ampicilina + sulbactam & 5 & 1,52 \\
\hline Tigeciclina & 2 & 0,6 \\
\hline Linezolida & 1 & 0,3 \\
\hline TOTAL & $\mathbf{3 2 9}$ & $\mathbf{1 0 0}$ \\
\hline Classe dos Antimicrobianos & 117 & 35,57 \\
\hline Carbapenêmico & 92 & 27,97 \\
\hline Glicopeptídeos & 70 & 21,28 \\
\hline Penicilinas & 24 & 7,29 \\
\hline Polimixinas & 14 & 4,26 \\
\hline Cefalosporinas & 9 & 2,73 \\
\hline Macrolídeos & 2 & 0,6 \\
\hline Glicilcilina & 1 & 0,3 \\
\hline Oxazolidinona & $\mathbf{3 2 9}$ & $\mathbf{1 0 0}$ \\
\hline TOTAL & 5 & \\
\hline
\end{tabular}

Fonte: Autores (2021).

A Tabela 3 mostra os diagnósticos presuntivo por grupos de doenças (CID-10) que justificaram direta ou indiretamente o uso de antimicrobiano, segundo os prescritores, o uso dos antimicrobianos. A maior justificativa utilizada, $89(27,05 \%)$ foi a ampliação do tratamento por indicar que os pacientes apresentaram leucocitose ou piora clínica. A sepse ou choque séptico foi 
a segunda maior justificativa, com 88 (26,75\%) do total de solicitações. Os pacientes que apresentaram Pneumonia comunitária ou nosocomial, Pneumonia associada a ventilação (PAV), Síndrome da Angustia Respiratória do Adulto (SARA) e Doença Pulmonar Obstrutiva Crônica (DPOC) foram categorizadas como Problemas do trato respiratório, sendo estes responsáveis por $77(23,40 \%)$ das solicitações dos antimicrobianos da UTI no período analisado.

Tabela 3. Diagnóstico presuntivo por grupos de doenças (CID-10) que justificaram direta ou indiretamente o uso de antimicrobiano UTI - Lagarto, 2019.

\begin{tabular}{lcc}
\hline & $\mathbf{n}$ & $\mathbf{\%}$ \\
\hline Ampliação do tratamento (Leucocitose ou piora clínica) & 89 & 27,05 \\
\hline Sepse e choque séptico & 88 & 26,75 \\
\hline Problemas do trato respiratório & 77 & 23,40 \\
\hline Neutropenia febril & 14 & 4,26 \\
\hline Síndrome de fournier & 8 & 2,43 \\
\hline Bactéria multirresistente (KPC, E. coli, Pseudomonas, Acinetobacter) & 2,13 \\
\hline Fístula enterocutânea (Colite pseudomembranosa) & 7 & 2,13 \\
\hline Descalonamento / Ajuste renal & 7 & 2,13 \\
\hline Abdome agudo traumático e infecção intra abdominal & 7 & 1,82 \\
\hline Infecção do trato urinário & 6 & 1,52 \\
\hline Meningite & 5 & 1,52 \\
\hline Celulite & 5 & 1,22 \\
\hline Infecção associada ao cateter venoso central (bacteremia) & 4 & 0,91 \\
\hline Osteomielite & 3 & 0,91 \\
\hline Escara infectada & 3 & 0,30 \\
\hline Diarreia causada por Clostridium difficile & 1 & 0,30 \\
\hline Fratura exposta calcânea - pé diabético & 1 & 0,30 \\
\hline Complicações HIV/AIDS (proteção para bactéria atípica) & 1 & 0,30 \\
\hline Endocardite infeciosa & 1 & 0,30 \\
\hline Secreção vaginal não especificada & 1 & 0,30 \\
\hline TOTAL & 1 & $\mathbf{1 0 0}$ \\
\hline
\end{tabular}

Fonte: Autores (2021).

Situações clínicas como lesões por pressão infectadas, fratura exposta, diarreia causada por Clostridium difficile, Complicações da Síndrome da Imunodeficiência Adquirida (HIV/AIDS), endocardite e secreção vaginal não especificada, foram as justificativas que menos se apresentaram, cada uma indicada por apenas uma solicitação.

Nas solicitações analisadas, 328 (99,7\%) dos antimicrobianos estavam prescritos na forma farmacêutica injetável para serem administrados por via intravenosa e apenas $1(0,3 \%)$ estava na forma farmacêutica injetável para ser utilizada por via oral, o que caracterizava um uso off label do medicamento vancomicina para tratamento de diarreia causada por Clostridium difficile.

A respeito das avaliações dos infectologistas sobre as solicitações de antimicrobianos de uso restrito, $223(67,78 \%)$ das solicitações foram aceitas sem qualquer alteração do infectologista diante da prescrição do médico assistencial. 88 (26,75\%) das solicitações foram autorizadas, mas com alterações realizadas pelos infectologistas e intervenções da equipe da farmácia. Foi constatado que apenas $18(5,47 \%)$ destas não foram autorizadas (Tabela 4). 
Tabela 4. Autorização pelo Infectologista e Avaliação farmacêutica.

\begin{tabular}{lcc}
\hline & $\mathbf{n}$ & $\boldsymbol{\%}$ \\
\hline Sim & 223 & 67,78 \\
\hline Sim com alteração & 88 & 26,75 \\
\hline Não & 18 & 5,47 \\
\hline TOTAL & $\mathbf{3 2 9}$ & $\mathbf{1 0 0}$ \\
\hline
\end{tabular}

Fonte: Autores (2021).

O tempo médio de tratamento solicitado inicialmente foi de 9,24 dias. Após a revisão e autorização pelos médicos infectologistas e farmacêuticos, o tempo médio de tratamento caiu para 8,92 dias, com valores limites de 2 a 14 dias.

A Tabela 5 mostra a Dose Diária Definida de cada antimicrobiano de uso restrito utilizado na Unidade de Terapia Intensiva.

Tabela 5. Dose Diária Definida (DDD) dos Antimicrobianos de Uso Restrito UTI - Lagarto, 2019.

\begin{tabular}{|c|c|c|c|c|c|c|c|c|}
\hline \multirow{2}{*}{$\begin{array}{c}2019 \\
\text { Mês }\end{array}$} & \multicolumn{2}{|c|}{$\begin{array}{c}\text { Ampicilina + } \\
\text { Sulbactam }\end{array}$} & \multicolumn{2}{|c|}{ Cefepime } & \multicolumn{2}{|c|}{ Meropenem } & \multicolumn{2}{|c|}{ Teicoplanina } \\
\hline & $\begin{array}{l}\text { Valor } \\
\text { mensal }\end{array}$ & $\begin{array}{l}\text { Valor } \\
\text { diário }\end{array}$ & Valor mensal & $\begin{array}{l}\text { Valor } \\
\text { diário }\end{array}$ & $\begin{array}{l}\text { Valor } \\
\text { mensal }\end{array}$ & $\begin{array}{l}\text { Valor } \\
\text { diário }\end{array}$ & $\begin{array}{l}\text { Valor } \\
\text { mensal }\end{array}$ & Valor diário \\
\hline Janeiro & 0 & 0 & $156,78 \mathrm{~g}$ & $5,05 \mathrm{~g}$ & $739,41 \mathrm{~g}$ & $\begin{array}{c}23,85 \\
\mathrm{~g}\end{array}$ & 0 & 0 \\
\hline Fevereiro & $50,85 \mathrm{~g}$ & $1,42 \mathrm{~g}$ & $67,80 \mathrm{~g}$ & $2,42 \mathrm{~g}$ & $425,85 \mathrm{~g}$ & $\begin{array}{c}15,20 \\
\mathrm{~g} \\
\end{array}$ & $21,19 \mathrm{~g}$ & $0,75 \mathrm{~g}$ \\
\hline Março & $50,85 \mathrm{~g}$ & $1,64 \mathrm{~g}$ & $127,12 \mathrm{~g}$ & $4,10 \mathrm{~g}$ & & $\begin{array}{c}18,69 \\
\mathrm{~g} \\
\end{array}$ & & 0 \\
\hline Abril & 0 & 0 & $38,14 \mathrm{~g}$ & $1,27 \mathrm{~g}$ & $577,33 \mathrm{~g}$ & $\begin{array}{c}19,24 \\
\mathrm{~g}\end{array}$ & 0 & 0 \\
\hline Maio & 0 & 0 & $25,64 \mathrm{~g}$ & $0,82 \mathrm{~g}$ & $323,72 \mathrm{~g}$ & $\begin{array}{c}10,44 \\
\mathrm{~g}\end{array}$ & $\begin{array}{c}132,48 \\
\mathrm{~g}\end{array}$ & $4,27 \mathrm{~g}$ \\
\hline Junho & 0 & 0 & $354,77 \mathrm{~g}$ & $\begin{array}{c}11,82 \\
\mathrm{~g} \\
\end{array}$ & $536,31 \mathrm{~g}$ & $\begin{array}{c}17,87 \\
\mathrm{~g} \\
\end{array}$ & $\begin{array}{c}178,42 \\
\mathrm{~g}\end{array}$ & $5,94 \mathrm{~g}$ \\
\hline Julho & $421,05 \mathrm{~g}$ & $\begin{array}{c}13,58 \\
\mathrm{~g}\end{array}$ & $178,95 \mathrm{~g}$ & $5,77 \mathrm{~g}$ & $280,70 \mathrm{~g}$ & $9,05 \mathrm{~g}$ & $66,67 \mathrm{~g}$ & $2,15 \mathrm{~g}$ \\
\hline Agosto & $219,51 \mathrm{~g}$ & $7,08 \mathrm{~g}$ & $62,72 \mathrm{~g}$ & $2,02 \mathrm{~g}$ & $452,09 \mathrm{~g}$ & $\begin{array}{c}14,58 \\
\mathrm{~g}\end{array}$ & $80,14 \mathrm{~g}$ & $2,58 \mathrm{~g}$ \\
\hline Setembro & $167,25 \mathrm{~g}$ & $5,57 \mathrm{~g}$ & $73,17 \mathrm{~g}$ & $2,43 \mathrm{~g}$ & $524,39 \mathrm{~g}$ & $\begin{array}{c}16,91 \\
\mathrm{~g} \\
\end{array}$ & $\begin{array}{c}149,83 \\
\mathrm{~g}\end{array}$ & $4,99 \mathrm{~g}$ \\
\hline Outubro & $257,84 \mathrm{~g}$ & $8,31 \mathrm{~g}$ & $99,30 \mathrm{~g}$ & $3,20 \mathrm{~g}$ & $733,45 \mathrm{~g}$ & $\begin{array}{c}23,65 \\
\mathrm{~g} \\
\end{array}$ & $\begin{array}{c}263,07 \\
\mathrm{~g}\end{array}$ & $8,48 \mathrm{~g}$ \\
\hline Novembro & $251,75 \mathrm{~g}$ & $8,39 \mathrm{~g}$ & $31,47 \mathrm{~g}$ & $1,04 \mathrm{~g}$ & $302,45 \mathrm{~g}$ & $\begin{array}{c}10,08 \\
\mathrm{~g}\end{array}$ & $45,45 \mathrm{~g}$ & $1,51 \mathrm{~g}$ \\
\hline Dezembro & $597,51 \mathrm{~g}$ & $\begin{array}{c}19,27 \\
\mathrm{~g}\end{array}$ & 0 & 0 & $577,80 \mathrm{~g}$ & $\begin{array}{c}18,63 \\
\mathrm{~g}\end{array}$ & 116,18 & $3,74 \mathrm{~g}$ \\
\hline 2019 & \multicolumn{2}{|c|}{$\begin{array}{c}\text { Piperacilina + } \\
\text { Tazobactam }\end{array}$} & \multicolumn{2}{|c|}{ Vancomicina } & \multicolumn{2}{|c|}{ Polimixina } & \multicolumn{2}{|c|}{ Linezolida } \\
\hline Mês & $\begin{array}{c}\text { Valor } \\
\text { mensal }\end{array}$ & $\begin{array}{l}\text { Valor } \\
\text { diário }\end{array}$ & Valor mensal & $\begin{array}{l}\text { Valor } \\
\text { diário }\end{array}$ & $\begin{array}{c}\text { Valor } \\
\text { mensal }\end{array}$ & $\begin{array}{l}\text { Valor } \\
\text { diário }\end{array}$ & $\begin{array}{c}\text { Valor } \\
\text { mensal }\end{array}$ & Valor diário \\
\hline Janeiro & $234,26 \mathrm{~g}$ & $7,55 \mathrm{~g}$ & $213,98 \mathrm{~g}$ & $6,90 \mathrm{~g}$ & 0 & 0 & 0 & 0 \\
\hline Fevereiro & $186,59 \mathrm{~g}$ & $6,66 \mathrm{~g}$ & $169,49 \mathrm{~g}$ & $6,05 \mathrm{~g}$ & $33,90 \mathrm{~g}$ & $1,21 \mathrm{~g}$ & 0 & 0 \\
\hline Março & $32,69 \mathrm{~g}$ & $1,05 \mathrm{~g}$ & $275,42 \mathrm{~g}$ & $8,88 \mathrm{~g}$ & 0 & 0 & 0 & 0 \\
\hline Abril & $147,09 \mathrm{~g}$ & $4,90 \mathrm{~g}$ & $158,90 \mathrm{~g}$ & $5,29 \mathrm{~g}$ & 0 & 0 & 0 & 0 \\
\hline Maio & $188,19 \mathrm{~g}$ & $6,07 \mathrm{~g}$ & $33,12 \mathrm{~g}$ & $1,06 \mathrm{~g}$ & 111,11 & $3,58 \mathrm{~g}$ & 0 & 0 \\
\hline Junho & $90,69 \mathrm{~g}$ & $3,02 \mathrm{~g}$ & $80,91 \mathrm{~g}$ & $2,69 \mathrm{~g}$ & 28,22 & $0,91 \mathrm{~g}$ & 0 & 0 \\
\hline Julho & $270,11 \mathrm{~g}$ & $8,71 \mathrm{~g}$ & $140,35 \mathrm{~g}$ & $4,52 \mathrm{~g}$ & $129,82 \mathrm{~g}$ & $4,18 \mathrm{~g}$ & $38,60 \mathrm{~g}$ & $1,24 \mathrm{~g}$ \\
\hline
\end{tabular}




\begin{tabular}{c|cc|cc|cc|cc}
\hline Agosto & $314,15 \mathrm{~g}$ & $\begin{array}{c}10,13 \\
\mathrm{~g}\end{array}$ & $123,69 \mathrm{~g}$ & $3,99 \mathrm{~g}$ & 223,00 & $7,19 \mathrm{~g}$ & 0 & 0 \\
\hline Setembro & $145,59 \mathrm{~g}$ & $4,85 \mathrm{~g}$ & $120,21 \mathrm{~g}$ & $4 \mathrm{~g}$ & $41,81 \mathrm{~g}$ & $1,39 \mathrm{~g}$ & 0 & 0 \\
\hline Outubro & $122,64 \mathrm{~g}$ & $3,95 \mathrm{~g}$ & $95,82 \mathrm{~g}$ & $3,09 \mathrm{~g}$ & $874,56 \mathrm{~g}$ & $\begin{array}{c}28,21 \\
\mathrm{~g}\end{array}$ & 0 & 0 \\
\hline Novembro & 118,57 & $3,95 \mathrm{~g}$ & $127,62 \mathrm{~g}$ & $4,25 \mathrm{~g}$ & 167,83 & $5,59 \mathrm{~g}$ & 0 & 0 \\
\hline Dezembro & 112,03 & $3,61 \mathrm{~g}$ & 182,57 & $5,88 \mathrm{~g}$ & $448,13 \mathrm{~g}$ & $\begin{array}{c}14,45 \\
\mathrm{~g}\end{array}$ & 0 & 0 \\
\hline
\end{tabular}

Fonte: Autores (2021).

\section{Discussão}

As medidas de restrição do uso de antimicrobianos têm aumentado em todo o mundo devido a crescente expansão da resistência bacteriana e a diminuição de desenvolvimento de novos fármacos voltados para o combate desses microrganismos (Carneiro, 2011 \& Casaes, 2019).

A resistência se apoia principalmente no uso inadequado dos antimicrobianos, configurando as principais causas a automedicação, erros de administração relacionados à duração do tratamento e a posologia o que pode gerar um aumento no tempo e os custos da hospitalização, além de elevar o risco de mortalidade (Lima Júnior, 2019 \& Carvalho, 2021).

No ambiente hospitalar, de maneira peculiar, as precauções quanto ao uso de antimicrobianos devem ser ainda maiores, devido à grande prevalência do uso desses. O ideal é que a terapia medicamentosa seja instituída com base em exames e aspectos clínicos dos pacientes. E que em casos graves e na ausência desses exames seja realizado o uso precoce de antimicrobianos de amplo espectro com cautela, considerando posterior descalonamento (Casaes, 2019 \& Carvalho, 2021).

O descalonamento de antimicrobianos é a diminuição do espectro ou do tempo do medicamento utilizado, devendo esta ação ser direcionada pela sensibilidade do microrganismo por diminuir a chance de ocorrer a resistência bacteriana. Deve ser feito assim que possível, observando a condição do paciente, quais os microrganismos identificados na cultura e a sensibilidade do antibiograma. (Moraes et al, 2016). Este estudo identificou apenas 7 (2,13\%) das solicitações que utilizavam como justificativa o descalonamento de antimicrobianos.

Ao analisar os resultados percebeu-se uma pequena diferença entre a utilização dos antimicrobianos de acordo o sexo, sendo o masculino ligeiramente superior no número de solicitações com 52,8\%. Um estudo realizado em um hospital de ensino por Santos et al. (2016) mostra dados parecidos sobre a prevalência do sexo masculino, mas com uma margem superior de diferença quando comparado a este trabalho. Outro trabalho realizado Silva (2012) em um hospital público do Pará também demonstrou um número maior do sexo masculino.

Houve variação na idade dos pacientes, mas os idosos representam a maior parte, com 41,51\% das solicitações. Souza (2015) explica este fator pelas alterações sofridas no organismo ao longo dos anos no processo de envelhecimento, que afetam inclusive o sistema imunológico, tornando o indivíduo suscetível a infecções oportunistas e necessitado do uso de antimicrobianos, sendo ainda constatado que o tempo e o gasto decorrente da internação dessa faixa etária são superiores em relação as outras. Um outro estudo realizado por De Souza et al. (2017) em uma Unidade de Terapia Intensiva de um hospital público, também demostrou essa faixa etária como a mais prevalente, sendo que dos 304 pacientes que participaram da pesquisa, 225 tinham idade superior há 75 anos.

Considerando a Política Nacional do Idoso, os idosos são pessoas com idade igual ou superior a 60 anos. Já Organização Mundial da Saúde define o idoso como pessoa com 60 anos ou mais, em país em desenvolvimento, e em países desenvolvidos os idosos são definidos como pessoas que apresentam 65 anos ou mais. (De Souza et al., 2017)

É importante salientar que cada vez mais os Estudos de Utilização de Medicamentos (EUM) têm sido considerados como importante ferramenta para garantir maior segurança do uso racional de medicamentos, planejar e gerenciar os serviços de 
Assistência Farmacêutica, nortear as políticas de medicamentos e subsidiar as políticas de saúde. Isto porque os medicamentos ocupam atualmente um lugar importante nos sistemas de saúde, tanto no aspecto econômico, quanto nos aspectos técnicos (resolutividade dos serviços) e sociais (uso racional de medicamentos) (Leite, 2008).

O medicamento mais solicitado foi o carbapenêmico meropenem. Este dado é controverso se comparado ao estudo de Dantas et al. (2015) realizado em um hospital universitário e que também analisava os antimicrobianos de uso restrito, pois a classe de medicamento mais solicitada foram as cefalosporinas, sendo que os carbapenêmicos estavam relacionados aos ajustes dos tratamentos realizados pelos avaliadores das solicitações.

Segundo De Souza et al. (2017) o alto uso do meropenem pode ser explicado pelo fato dele ser um antimicrobiano de amplo espectro, utilizado inicialmente como terapia empírica em situações consideradas graves, e depois mantido como meio de se ter mais segurança e uma ampla efetividade contra diversos microrganismos.

Ainda assim, a utilização dessa classe deveria ser menos frequente que a encontrada nesse estudo, pois podem causar resistência da Pseudomonas Aeruginosa, da Acinetobacter baumanni, e Klebsiella Pneumoniae Carbapenemase (KPC), sendo microrganismos que adquirem resistência aos antimicrobianos produtoras de enzimas que degradam os medicamentos, e que estão correlacionadas ao aumento das infecções e da morbimortalidade, se tornando um dos maiores desafios para o controle de infecções ocorridas em Unidades de Terapia Intensiva (Santos, 2017).

Percebeu-se durante a realização da coleta de dados, que existia pouca atuação diária do profissional farmacêutico clínico no controle dos antimicrobianos de uso restrito, pois o fluxo do setor estabelece apenas o recebimento da solicitação do medicamento, a alimentação de uma tabela e posterior impressão desta, que é utilizada como guia na dispensação das prescrições. Os infectologistas do hospital são os responsáveis pela avaliação da prescrição, e posterior autorização ou negação do tratamento solicitado.

Os residentes em farmácia eram os profissionais do setor de farmácia com maior contato com o paciente e, apesar do revezamento entre os setores do hospital, quando estavam presentes na UTI realizavam intervenções sobre os antimicrobianos, sua dosagem, tempo de tratamento, ajuste de dose, entre outros.

Dentre as atribuições do profissional farmacêutico em unidades hospitalares está a avaliação das prescrições médicas na identificação de possíveis interações medicamentos, erros de medicação relacionados a vias de administração e doses, acompanhamento de eventos adversos e alergias, dentre outras. Ressalta-se assim que a função do profissional vai além das atividades administrativas, pois as novas tendências da área são direcionadas diretamente ao paciente, buscando fornecer uma assistência técnica, mas também clínica. Nos casos de antibioticoterapia o profissional deve interagir com os prescritores a fim de garantir a racionalidade e eficácia dos medicamentos, pois estes tendem a aumentar o risco de resistência bacteriana (Santos, 2019).

O farmacêutico como profissional participante da Comissão de Controle de Infecção Hospitalar (CCIH) possui a responsabilidade de avaliar as prescrições e, juntamente com uma equipe multidisciplinar, elaborar protocolo da unidade e indicadores, facilitam a padronização dos medicamentos no hospital (De Sousa Feitosa, 2019). Pode ainda realizar análise de antibiograma, realizar a atenção farmacêutica estimulando o controle a terapia, elaborar relatórios sobre o consumo desses medicamentos e propor treinamentos que visem evitar a disseminação de microrganismos (Rodrigues, 2010).

Um estudo realizado em um hospital público avaliou anteriormente e posteriormente o impacto da racionalização do uso de antimicrobianos realizada pela farmácia e a contribuição da CCIH. Observou-se nesse período o aumento do consumo de penicilinas e cefalosporinas, mas em contrapartida uma diminuição significativa de uso dos aminoglicosídeos, carbapenêmicos e quinolonas, que segundo alguns autores são grandes responsáveis por reações adversas e resistência bacteriana. (Franco, 2015) 
Os meses de julho, agosto e dezembro foram os com maiores índices de solicitações, valendo ressaltar que julho e agosto são os meses mais frios da região e seguintes ao período festivo junino, amplamente comemorado na região com fogos de artifícios e fogueira, podendo este ser um fator de agravamento ou gatilho para diversos quadros respiratórios.

O Organização Mundial de Saúde (OMS) orienta a utilização de indicadores que possam mensurar o consumo dos antimicrobianos, entre eles a Dose Diária Definida (DDD). Esse método define a dose diária média do consumo de determinado fármaco na sua mais importante indicação (Feitosa; Assis \& Coêlho, 2018).

A vantagem mais significativa do uso de DDD é que se torna possível a comparação de resultados entre países e através de uma linha temporal, sem que haja comprometimento dos dados devido preço e apresentação dos medicamentos que podem mudar. Um ponto negativo é o fato de que essa dose nem sempre é equivalente a dose prescrita e nem mesmo a dose média utilizada (Melo; Ribeiro \& Storpirtis, 2006).

\section{Considerações Finais}

Foram identificados os medicamentos mais prescritos e a sua relação com as patologias apresentadas, sendo o Meropenem, Vancomicina e a associação de Piperacilina com Tazobactam são os mais prevalentes entre os antimicrobianos de uso restrito, como visto em outros estudos, devido seu amplo espectro e pelo fato do hospital não realizar em suas dependências os testes de cultura, levando a uma maior ocorrência de tratamento empírico. A sepse e as infecções do trato respiratório foram as condições clínicas de maior prevalência nos pacientes, sendo alguns dos principais problemas enfrentados nas unidades hospitalares.

A partir desses resultados pode-se gerar indicadores que norteiem acerca da melhora da assistência prestada na unidade, evidenciando através do perfil das prescrições dos antimicrobianos, as discrepâncias mais recorrentes e os acertos. Com isso, estabelecer um diagnóstico situacional epidemiológico da unidade e possibilitar a criação de um Programa de gerenciamento para o uso racional de antimicrobianos - Antimicrobial Stewardship. Pode ainda suscitar no setor de Farmácia Hospitalar a importância da maior atuação do farmacêutico clínico junto a Comissão de Controle à Infecção Hospitalar (CCIH), visando o melhoramento dos fluxos de dispensação e controle dos antimicrobianos e podendo assim, minimizar o desenvolvimento de potenciais resistências bacterianas.

Este irá subsidiar indicadores epidemiológicos que poderão ser utilizados para a melhoria da assistência à saúde, podendo interferir de maneira direta na eficácia de tratamentos propostos pelos prescritores, além de nortear sobre pontos que necessitam de ajuste no fluxo de prescrição e liberação de tais medicamentos.

\section{Referências}

Brasil. (2017). Ministério da Saúde. Agência Nacional de Vigilância Sanitária. Diretriz Nacional para Elaboração de Programa de Gerenciamento do Uso de Antimicrobianos em Serviços de Saúde.

Brasil. (2001). Ministério da Saúde. Agência Nacional de Vigilância Sanitária. Gerência-Geral de Tecnologia em Serviços de Saúde. Unidade de Controle de Infeccção em Serviços de Saúde. Consenso sobre o uso racional de antimicrobianos.

Carneiro, M. et al. (2011). O uso de antimicrobianos em um hospital de ensino: uma breve avaliação. Rev. Assoc. Med. Bras.; 57; $421-424$.

Carvalho, P. P., et al (2021). Evaluation of critical patients with suspected sepsis in a university hospital. Research, Society and Development, 10 , e39410313570.

Casaes, F. F. (2019). Descalonamento, adequação antimicrobiana e prevalência da prescrição de Piperacilina/Tazobactam em um hospital geral. Trabalho de Conclusão de curso - Universidade Federal da Fronteira Sul, Passo Fundo.

Dantas, J. O. et al. (2015). Avaliação da prescrição de antimicrobianos de uso restrito em um hospital universitário. Journal of Infection Control, 4, 39-48.

De Souza, F. C., Baroni, M. M. F., \& Roese, F. M. (2017). Perfil de utilização de antimicrobianos na unidade de terapia intensiva de um hospital público. Revista Brasileira de Farmácia Hospitalar e Serviços de Saúde 
Research, Society and Development, v. 10, n. 8, e15710816565, 2021

(CC BY 4.0) | ISSN 2525-3409 | DOI: http://dx.doi.org/10.33448/rsd-v10i8.16565

Franco, J. M. P. L. et al. (2015). O papel do farmacêutico frente à resistência bacteriana ocasionada pelo uso irracional de antimicrobianos. Semana Acadêmica Revista Científica, 1, 1-17.

Leite, S. N., Vieira, M., \& Veber, A. P. (2008). Estudos de utilização de medicamentos: uma síntese de artigos publicados no Brasil e América Latina.

Lemos, G. S. et al. (2016). Prescrições de antimicrobianos de uso restrito de pacientes internados em um hospital de ensino. Rev. Bras. Farm. Hosp. Serv. Saúde $7 ; 8-12$.

Lima Júnior, F. A. et al. (2019). Perfil das prescrições de antibioticoterapia em uma unidade de terapia intensiva adulto de um hospital de referência cirúrgica no estado do maranhão. Revista Eletrônica Acervo Saúde, e1301-e1301.

Mata, A. P. T. G., \& Da Silva, L. L. (2011). Controle de infecção hospitalar em unidade de terapia intensiva: estudo retrospectivo. Semina: Ciências Biológicas e da Saúde; 32; 47-58.

Mota, L. M. et al. (2010). Uso racional de antimicrobianos. Medicina (Ribeirao Preto. Online); 43; 164-172.

Moraes, R. B. et al. (2016). Descalonamento, adequação antimicrobiana e positividade de culturas em pacientes sépticos: estudo observacional. Rev. Bras. Ter. Intensiva, 28, 315-322.

Santos, R. G. et al. (2016). Prescrições de antimicrobianos de uso restrito de pacientes internados em um hospital de ensino. Rev. Bras. Farm. Hosp. Serv; 7; 812 .

Santos, E. B., \& Pereira, K. M. (2017). Estudo do uso de carbapenémicos em uma unidade de terapia intensiva adulta de um hospital público do município de Vitória da Conquista-Bahia. Revista De Psicologia, 11, 188-198.

Santos, L. Q. (2017). Uso racional de antimicrobianos e no ambiente hospitalar. Journal Of Biology \& Pharmacy And Agricultural Management

Silva, E. R. M. (2012). Análise do perfil das prescrições de antimicrobianos na clínica médica de um hospital público do Pará. Revista Brasileira De Farmácia Hospitalar E Serviços De Saúde

Sousa, K. C., et al. (2015). Tendências de prescrição de antimicrobianos em idosos hospitalizados em um hospital universitário. Saúde e Pesquisa, 8, 501-508.

Rodrigues, F. D'a., \& Bertoldi, A. D. (2010). Perfil da utilização de antimicrobianos em um hospital privado. Ciênc. saúde coletiva; 15; $1239-1247$.

World Health Organization - WHO. (1985). Rational use of drugs: Report of conference of experts. Nairobi.

De Sousa Feitosa, T, Da Silva Assis, R. A., \& Coêlho, M. L. (2018). Utilidade de indicadores para o monitoramento do consumo de antimicrobianos de uso restrito em uma Unidade De Terapia Intensiva. Jornal de Ciências da Saúde do Hospital Universitário da Universidade Federal do Piauí, 1, $42-50$. 